\title{
Vereinfachte Emesis-Prävention mit Fosaprepitant
}

Die antiemetische Wirksamkeit der i.v. Einmalgabe von 150mg Fosaprepitant in Kombination mit Ondansetron/Dexamethason ist mindestens vergleichbar mit der einer entsprechenden Kombination mit 3-maliger p.o. Gabe von Aprepitant. In der doppelblinden, randomisierten Phase-III-Studie EASE (Evaluation of Fosaprepitant in Single-dose Schedule)-017 konnte die Nicht-Unterlegenheit der Fosaprepitant-haltigen Antiemese bei Patienten, die erstmals mit einer Cisplatin-basierten Chemotherapie mit $\geq 70 \mathrm{mg} / \mathrm{m}^{2} \mathrm{KOF}$ Cisplatin behandelt wurden, gezeigt werden (Grunberg SM et al., ASCO 2010: \#9021).

In die Analyse gingen die Daten von 2.247 Patienten ein, die randomisiert an Tag 1 eine i.v. Einmalgabe von Fosaprepitant (150mg) oder für 3 Tage p.o. Aprepitant (125mg Tag 1, 80mg Tage 2, 3) erhielten. In beiden Gruppen wurden zur Emesis-Prävention zusätzlich i.v. Ondansetron (jeweils 32mg Tag 1) und p.o. Dexamethason (jeweils 12mg Tag 1; Fosaprepitant-Gruppe 8mg Tag 2, 16mg Tage 3, 4; Aprepitant-Gruppe: 8mg Tage 2-4) gegeben. Im primären Endpunkt, komplettes Ansprechen (kein Erbrechen, keine Bedarfsmedikation in den ers- ten 120 Stunden nach Beginn der Chemotherapie) konnte die Nicht-Unterlegenheit des Fosaprepitanthaltigen Regimes im Vergleich zur Dreierkombination mit Aprepitant gezeigt werden (Tab.). Die Verträglichkeit beider Schemata war insgesamt vergleichbar.

Diese Studienergebnisse zeigen eine Option für eine vereinfachte Emesis-Prä-

Endpunkt

\section{Antiemetische Wirksamkeit}

Fosaprepitant-

Regime

$(n=1.138)$

Aprepitant-

Regime

$(n=1.109)$

komplettes Ansprechen $\quad 71,9 \% *$

$72,3 \%$

${ }^{*}$ Differenz-0,4\% (95-\%-Konfidenzintervall -4,1\%-3,3\%)

nach Grunberg SM et al., ASCO 2010: \#9021 and poster presentation

vention mit Neurokinin-1-Rezeptorantagonisten in Kombination mit einem Glucocorticoid und einem Serotonin-Antagonisten auf. $A M$

Hormonrefraktäres Prostatakarzinom

\section{Denosumab verzögert Skelettkomplikationen bei Knochenmetastasen}

Patienten mit hormonrefraktärem Prostatakarzinom und Knochenmetastasen können von der Therapie mit Denosumab besonders profitieren. Wie die Ergebnisse einer aktuellen Phase-III-Studie zeigen, verzögerte Denosumab die Zeit bis zum Auftreten von Skelettkomplikationen stärker und beugte Skelettkomplikationen besser vor als ein Standardbisphosphonat (Fizazi K et al., ASCO 2010: \#LBA4507).

Denosumab ist ein humaner gegen RANK (Receptor Activator of Nuclear Factor kappa B)-Ligand gerichteter Antikörper. RANK-Ligand ist ein wichtiger Regulator der Osteoklasten und ein zentraler Mediator im Circulus vitiosus der Knochenzerstörung bei metastasierten Tumorerkrankungen. Seit Mai 2010 ist Denosumab (Prolia ${ }^{\circledR}$ ) in der Europäischen Union u.a. zugelassen zur Behandlung von Knochenschwund im Zusammenhang mit einer Hormonablationstherapie bei Prostatakarzinom-Patienten mit erhöhtem Frakturrisiko. Mit dem RANK-Ligand-Antikörper kann bei diesen Patienten das Frakturrisiko deutlich reduziert werden.

Erste Studienergebnisse zum Einsatz von Denosumab bei Knochemetastasen z.B. bei Mammakarzinom-Patienten oder Patienten mit multiplem Myelom zeigen eine im Vergleich zur Behandlung mit Zoledronsäure mindestes gleichwertige Vorbeugung bzw. Verzögerung skelettaler Ereignisse (SRE=Skeletal Related Event).

\section{Monate länger komplikationsfrei}

Die doppelblinde Phase-III-Studie von Fizazi et al. schloss Patienten mit hormonrefraktärem Prostatakarzinom und Knochenmetastasen ein, die randomisiert Denosumab (120mg s.c. + Placebo i.v. alle 4 Wochen; $n=950$ ) oder Zoledronsäure (4mg i.v. + Placebo s.c. alle 4 Wochen; $n=951$ ) erhielten. Mit einer Risikoreduktion um 18\% war die Therapie mit Denosumab der Therapie mit Zoledronsäure im primären Endpunkt, der Zeit bis zum ersten SRE, überlegen (Hazard-Ratio [HR] 0,82; $p=0,008$ für Überlegenheit). Das erste SRE trat mit dem Bisphosphonat nach median 17,1 Monaten, mit Denosumab nach median 20,7 Monaten ein, so Karim Fizazi, Villejuif/Frankreich. Auch das Risiko für das erste und nachfolgende SRE wurde um $18 \%$ reduziert (HR 0,82; $p=0,008)$. Die Nebenwirkungsraten waren in den Studienarmen ähnlich. AkutePhase-Reaktionen traten unter Zoledronsäure häufiger auf als unter Denosumab (17,8\% vs. 8,4\%). Hypokalzämien wurden unter Denosumab öfter beobachtet (12,8\% vs. 5,8\%). Die Inzidenz an Kieferosteonekrosen war insgesamt niedrig und in den Studienarmen ähnlich (2,3\% mit Denosumab vs. 1,3\% mit Zoledronsäure).

Als günstig wertete Fizazi die einfache Handhabung des Antikörpers im Klinikalltag: Renales Monitoring oder Dosismodifikationen bei eingeschränkter Nierenfunktion sind nicht erforderlich, ebenso entfällt das Management von Akute-Phase-Reaktionen. $K A$ 